CATARACT

\title{
Risk stratification for the humble cataract
}

\section{Liu}

\section{A chance to look at surgeon statistics, training, and ophthalmic surgical competence}

C ataract surgery has received more than its fair share of controversy. Senior figures in medicine, both ophthalmological and non-ophthalmological, have referred to cataract surgery as minor surgery, and advocated the deployment of non-medically qualified personnel. But "minor op" it certainly is not. There is a minute margin of error, with an anterior chamber volume of only $0.25 \mathrm{ml}$, the depth of which separates non-regenerating corneal endothelium, and the posterior capsule, which is only micrometres in thickness. Damage either at your own peril. "Experts" who worked on the Relative Values Review comparing how to remunerate surgeons working in different fields in the private sector should have taken this into consideration, and not how much time and physical work is involved.

In this issue of the BJO ( $p$ 1242), Muhtaseb et al found that over $40 \%$ of their cataract workload contained one or more risk factors. This was probably an underestimate with some risk factors not scored. These cases are more difficult to do and have a higher risk of developing operative complications. But even a routine case with a fully dilating pupil, crystal clear cornea with huge endothelial reserve, moderate nuclear hardness, a cooperative patient, and no problem with the first eye operation can go wrong. Patients often compare cataract surgery with a tooth extraction. They need to be reminded of the difference between the devastation of infective endophthalmitis and a tooth socket infection. In any case there are 32 teeth but only two eyes (in some cases only one)!

What is risk stratification? Basically, not all cases are the same and some (higher risk) cases will be more prone to develop complications because of the technical difficulty and/or structural weakness of tissues (for example, the pseudoexfoliation cataract with poorly dilating pupil, weak zonules, and association with glaucoma). In other words, a cataract is not a cataract is not a cataract. Cardiac surgeons have understood the importance of risk stratification for many years.

There are a number of reasons why surgery should be risk stratified. It is only through knowing the risk of any particular case that meaningful preoperative counselling could take place, with mention of hospital or, even better, surgeon statistics for the particular risk group. Then there is the business of team building for high risk complex cases (see table 1 for cataract surgery). Low risk cases should be used for training novice surgeons, whereas graded higher risk cases are for advance training. Without knowing the case mix, surgeons' individual statistics as well as hospital statistics could not be compared with other surgeons and hospitals. Both in the NHS and in the private sector, one could argue for higher reimbursement for more complex cases. Finally, it could be argued that (complex) cataract surgery is in itself a subspecialty within ophthalmology.

Case mix in cataract surgery has become even more important in this decade as there is a trend to cream off routine cases for waiting list initiatives and for treatment centres. These routine cases will take less time to carry out. Surgeons operating on these could be less competent and yet would still have good statistics. Traditional NHS providers are then left with more complex cases, not just from the point of view of the eye. This will have a deleterious effect on both hospital and surgeon statistics. The cost per case also will be higher (more time, increased use of more expensive devices, higher risk of complications requiring vitrectomy equipment, higher risk of retinal detachment and endophthalmitis requiring further admission and treatment, etc). Such cases could cost up to several thousand pounds each. Muhtaseb et al have shown that even experienced consultants are getting 30\% plus complications rate in their highest risk group. Surgical training of inexperienced residents will suffer, or patients will suffer as a result of this creaming off process, although master class surgical training may improve.

There are a number of pitfalls of statistics. Firstly, there is "rubbish (data) in, rubbish (statistics) out"-a sad fact of life. Probity is also sometimes called into question. Detailed risk stratification is essential for meaningful comparison between individual surgeons and between hospitals. But even if the above could be fully dealt with, individual surgeon statistics is never really just that. The results of cataract surgery is team dependent, from the quality of biometry, of pre-assessment, experience, calibre and harmony of ward and theatre staff, the standard of equipment, adequate maintenance of same, quality of devices, of cleaning and sterilisation, and so on. Furthermore, while it is easy to assign responsibility to the trainee if a complication has already occurred when the trainer takes over the operation; the situation is not clear when a trainee produces a leaky wound and cloudy cornea, then the trainer takes over and then goes on to break the posterior capsule. Thus, the same surgeon may have different statistics in different NHS hospitals and in private hospitals because of the above and difference in case mix. And it goes without saying that the best surgeons specialising in complex cases may have poor statistics compared with their less able peers.

Table 1 The complex cataract/patient

\begin{tabular}{ll} 
Corneal guttata & Posterior polar cataract \\
Corneal scars/opacity & Children \\
Glaucoma & Co-existing diabetic retinopathy \\
Pseudoexfoliation & Vitrectomised eye \\
Traumatic cataract & Scleritis \\
Subluxated lens & Bleeding disorders \\
Small pupil & High risk of expulsive haemorrhage \\
Nanophthalmos & Deep set eye \\
High myopia & Torticollis \\
Iritis & Chronic obstructive pulmonary disease \\
Mature cataract & Anxious patient \\
Hard nucleus & Deaf patient \\
Soft nucleus & Confused patient \\
\hline
\end{tabular}


What makes a good surgeon then? The process of professionalisation starts from entering medical school, or even earlier if there has been a role model in the family. The process continues throughout the many years of postgraduate training when the values of peers and mentors are absorbed by osmosis. The good surgeon comes to realise that patient interest is first and foremost. "First do no harm." The good surgeons hone their skills and keep up to date, as well as develop new techniques. However, surgery is not just a technical exercise. First, a surgeon learns how to operate; then when to operate. Finally they learn when not to operate. Competency of all three "degrees" is important. The balance of the second and the third is affected by the actual ability, confidence (self perceived ability), risk taking behaviour, and clinical maturity.

I would suggest the following list as negative markers of surgical (technical) competence. Imperfect wound architecture, iris damage, anterior capsulorrhexis tear, decentred anterior capsulorrhexis, posterior capsular rupture, vitreous loss, persistent corneal oedema, and endophthalmitis. The questions surgeons may wish to ask themselves after each case could be "Has full visual potential been realised?" "Has refractive aim been achieved, both spherical and astigmatic?" "Is the patient satisfied?" "Has there been a complication?" "If so, was the eye at particular risk of that complication?" "Have I done all that could be done to avoid that complication?"

Muhtaseb et al have made an excellent start with demonstrating that a simple scoring system works for predicting peroperative complications in cataract surgery. Simplicity is important as there is a universal dislike for form filling. The next step is to have the scoring system validated in other ophthalmic units. At the same time, a longer term multicentre project should be set up to work out the exact risk for each graded risk factor, as opposed to a simple weighted system based on instinct and intuition. Patient related (as opposed to eye related) risk factors should also be included in the study. There should then be adoption of a nationally agreed risk stratification assessment, which should be used for all hospital and national audits. We can then, finally, compare like with like. It is hoped that the Royal College of Ophthalmologists will lead this. It will then be possible to have a glimpse of whether there is a big spread in surgical ability among surgeons. While half of us will be below average, we will have to set a lower limit to identify any surgeons who perform poorly (or who do not care!).

Br J Ophthalmol 2004;88:1231-1232. doi: $10.1136 /$ bjo.2004.048207

Correspondence to: C Liu, Sussex Eye Hospital, Eastern Road, Brighton BN2 5BF, UK; CSCLiv@aol.com

\section{Frequency doubling technology perimetry for neuro-ophthalmological diseases}

\section{A Johnson}

\section{It will be beneficial to all eye care practitioners if investigations of a wide variety of ocular and neurological diseases are evaluated with FDT perimetry}

\begin{abstract}
n the past decade, there have been many advances in diagnostic test 1 procedures for evaluation of structural and functional changes affecting the visual pathways. Perimetry and visual field testing have undergone improvements that allow tests to be performed more efficiently and accurately, detect earlier signs of pathological changes, quantitatively evaluate progressive losses, and provide enhanced diagnostic information..$^{1-3}$ One of these emerging visual field test procedures is frequency doubling technology (FDT) perimetry, which has been shown to be effective in detecting visual field loss produced by glaucoma and other visual disorders, ${ }^{4}$ and may detect early perimetric changes before standard automated perimetry. ${ }^{5}$ Because of its clinical value and importance, an improved second generation of FDT instrumentation,
\end{abstract}

known as the Humphrey Matrix, has recently become available. ${ }^{6}$ This new device attests to the significance and success of FDT perimetry as a clinical diagnostic tool.

The emphasis of FDT perimetry has been directed towards the detection and evaluation of glaucomatous visual field loss, and some papers have alluded to FDT perimetry as a procedure to be used predominantly for glaucoma patients and individuals at risk of developing glaucoma. However, FDT perimetry, like most visual field test procedures, was designed to evaluate peripheral sensitivity loss produced by a variety of disorders affecting the visual system, including neuro-ophthalmological diseases. ${ }^{4-9}$ It is unfortunate that only a few investigators have reporting FDT findings for visual dysfunction other than glaucoma. In this view, it is most encouraging to observe the article by Girkin et al in this issue of the BJO (p 1274) on FDT perimetry in nonarteritic ischaemic optic neuropathy with altitudinal defects.

In their study, the authors of this investigation report that FDT perimetry, in comparison with standard automated perimetry, is able to provide very good detection and characterisation of visual field loss in non-arteritic ischaemic optic neuropathy. FDT perimetry reveals more extensive damage that standard automated perimetry for some cases, and demonstrates a strong correlation with structural optic disc changes. These findings provide important clinical information for practitioners and augment our existing knowledge pertaining to the clinical value of FDT perimetry. Additionally, this report indicates that FDT perimetry may be helpful in future investigations of this type for determining insights and added information about the pathophysiological basis for the damage resulting from non-arteritic ischaemic optic neuropathy.

FDT perimetry is a relatively new visual field test procedure that has been available for approximately 7 years, has demonstrated clinical value and usefulness, and has resulted in development of a new device (the Humphrey Matrix) that appears to have many enhanced features. It will be beneficial to all eye care practitioners if investigations of a wide variety of ocular and neurological diseases are evaluated with FDT perimetry. The study of FDT findings in patients with non-arteritic ischaemic optic neuropathy and altitudinal 
defects, as reported here, is an excellent example of this type of research. The authors are to be congratulated for conducting such a careful, well designed, and timely study. Through these and similar efforts we will gain a better understanding of the clinical capabilities and limitations of FDT perimetry, enhance our understanding of the underlying pathophysiological basis of visual pathway disorders, and obtain an improved awareness of the association between structural and functional damage produced by ocular and neurological diseases. Hopefully, this work may serve as a source of motivation and inspiration for other researchers to pursue these types of studies pertaining to the clinical utility of FDT perimetry.
Br J Ophthalmol 2004;88:1232-1233.

doi: $10.1136 /$ bjo. 2004.051748

Correspondence to: C A Johnson, Discoveries in Sight, Devers Eye Institute, Legacy Health Systems, 1225 NE Second Avenue, Portland, OR 97208, USA; cajohnson@

discoveriesinsight.org

\section{REFERENCES}

1 Johnson CA. New developments in perimetry. Chapter 18. In: Fingeret, Lewis, eds. Primary care of the glaucomas. New York, McGraw-Hill, 2000:271-94.

2 Johnson CA. Update on perimetry: new developments. Glaucoma in the New Millenium. Proceedings of the 49th Annual Symposium of the New Orleans Academy of Ophthalmology. The Hague: Kugler Publications, 2003:185-206.

3 Sample PA, Araie M, Chauhan BC, et al. Comparison of methods for measurement of function. In: Weinreb RN, Greve EL, eds.
Glaucoma diagnosis, structure and function. The Hague: Kugler Publications, 2004:70-7.

4 Anderson AJ, Johnson CA. Frequency-doubling technology perimetry. In: Sample PA, Girkin CA eds. Ophthalmol Clin N Am 2003:16:213-25.

5 Sample PA, Bosworth CF, Blumenthal EZ, et al. Visual function-specific perimetry for indirect comparison of different ganglion cell populations in glaucoma. Invest Ophthalmol Vis Sci 2000:41:1783-90.

6 Fingeret $M$, Johnson CA. The new Humphrey Matrix FDT. Optometric Management 2003:December:1-8.

7 Thomas D, Thomas R, Muliyil JP, ef al. Role of frequency doubling perimetry in detecting neuroophthalmic visual field defects. Am J Ophthalmol 2001;131:741-5.

8 Fujimoto N, Adachi-Usami E. Frequency doubling perimetry in resolved optic neuritis. Invest Ophthalmol Vis Sci 2000;41:2558-60.

9 Wall M, Neahring RK, Woodward KR. Sensitivity and specificity of frequency doubling perimetry in neuro-ophthalmic disorders: a comparison with conventional automated perimetry. Invest Ophthalmol Vis Sci 2002;43:1277-83. 